\title{
Drug-Induced Liver Injury and COVID-19 Infection: The Rules Remain the Same
}

\author{
Alexandre Olry ${ }^{1} \cdot$ Lucy Meunier $^{2} \cdot$ Bénédicte Délire $^{3} \cdot$ Dominique Larrey $^{2} \cdot$ Yves Horsmans $^{3} \cdot$ Hervé Le Louët $^{4}$
}

Published online: 8 June 2020

(c) Springer Nature Switzerland AG 2020

The pandemic infection by coronavirus 2019 (COVID-19) that spreading from China is generating a tremendous flow of information and one emerging point is the frequent association of COVID-19 infection with liver abnormalities. Studies from China showed an increase of transaminases and other liver enzymes in $14-53 \%$ of infected patients [1-4]. These abnormalities were generally mild with moderate increase in transaminase levels in symptomatic and/or severe forms of COVID-19 infection [3, 4]. The mechanisms are still mostly unknown. It may involve the role of the virus itself, the role of virus-induced inflammation, liver hypoxia (thrombotic context, severe acute respiratory syndrome) and/or drug-induced liver injury (including paracetamol misuse, anti-infectious agents, etc.). Several anti-infectious and anti-cancer drugs with previous marketing authorization are proposed either to inhibit the COVID-19 replication or to minimize the clinical consequences and pulmonary failure. This concerns many drugs alone or in association, including hydroxychloroquine associated or not with azithromycin, lopinavir/ritonavir associated or not with interferon beta, remdesivir, baricitinib, imatinib, darunavir and umifenovir [5], and their immediate availability has led to their dissemination in several countries as compassionate use outside of clinical trials [5].

Alexandre Olry

alexandre.olry@aphp.fr

1 Pharmacovigilance Department, Henri Mondor University Hospital (APHP), 51 Avenue du Maréchal de Lattre de Tassigny, Créteil, 94010 Paris, France

2 Liver Unit, Saint-Eloi Hospital, INSERM 1183, Montpellier School of Medicine, Montpellier, France

3 Department of Hepatogastroenterology, Cliniques Universitaires Saint-Luc, Université Catholique de Louvain (UCL), Brussels, Belgium

4 CIOMS, Head of Vigilance Department, Henri Mondor University Hospital (APHP), Paris, France
In this context, the REFHEPS (Réseau d'Étude Francophone de l'Hépatotoxicité des Produits de Santé), a European French-speaking study network focused on druginduced liver injury (DILI), would like to inform physicians that just within 2 weeks, four cases of lopinavir/ritonavir combination discontinuation were reported in COVID-19 positive patients because of suspected drug-induced hepatotoxicity (unpublished data).

The withdrawal was based on isolated transaminases rate perturbation $<5$ times the upper limit of normal (ULN) level without any clear evidence of relationship, especially chronologically regarding the introduction date. It concerned four males (mean age 60 years) hospitalized in intensive care units with comorbidities like arterial hypertension and obesity (mean BMI $32 \mathrm{~kg} / \mathrm{m}^{2}$ ) but not diabetes mellitus. The data issued from the French pharmacovigilance database are consistent with our observation and confirmed the trend of drug discontinuation based on a misuse of the DILI definition.

The REFHEPS* network currently includes France and Belgium and deals with liver injury related to chemical drugs but also to herbal medicines and nutraceuticals. It has been created within the framework of the COST collaboration (European Cooperation in Science and Technology) and its purpose is to promote knowledge about the hepatotoxicity of health products, allowing better management and monitoring of DILI.

Hence, the REFHEPS group reviews regularly the data regarding the potential hepatotoxicity of all the drugs that are tested for COVID-19 and briefly summarizes currently known data on drugs used as COVID-19 treatment below:

- Hydroxychloroquine is a possible but rare cause of idiosyncratic DILI [6].

- Azithromycin may cause idiosyncratic acute liver injury. The clinical presentation is a cholestatic hepatitis arising within 1-3 weeks after starting treatment. Azithromycin 
was also associated with hepatocellular injury, with a short latency (a few days) [7].

- All forms of interferon beta have been shown to cause hepatic injury, although most cases are asymptomatic and mild. Most of the time its presentation is transient with mild elevations in serum aminotransferase levels and serum alkaline phosphatase levels usually normal or minimally elevated [8].

- Lopinavir administration is associated with moderateto-severe elevations in serum aminotransferase levels (>5 $\times$ ULN) in $3 \%$ to $10 \%$. The pattern of serum enzyme elevations varies from hepatocellular to cholestatic or mixed [9].

- Ritonavir, used at low 'booster' doses, does not appear to increase the frequency or severity of serum enzyme elevations, and those that occur are usually asymptomatic and self-limited, resolving even with continuation of ritonavir. However, due to its enzymatic inhibitor properties, ritonavir can enhance the plasmatic level of co-administered drugs which then raises the risk of their hepatotoxicity [10].

- Currently, we have very low data about the potential hepatotoxicity of remdesivir. This drug is not reported to cause liver injury in LiverTox [6]. Considering its compassionate use in patients with COVID-19 infection and the frequency of liver dysfunction with this disease, the attribution of hepatotoxicity to remdesivir is challenging.

- Baricitinib is currently being assessed in clinical trials as an inhibitor of JAK-1 and 2 for the treatment of rheumatoid polyarthritis. Its use has been associated with mild and transient transaminases elevation. In the large clinical trials, transaminase values $>5 \times$ ULN occurred in $<1 \%$ of patients on baricitinib [11].

- Imatinib, currently approved in several blood cancers, was associated with common elevations in serum aminotransferase levels, but ALT levels $>5 \times$ ULN range occur in only $2-4 \%$ of patients treated for 6 months or more [12]. In addition, mild elevations in serum bilirubin can occur. These abnormalities are usually mild, asymptomatic and resolve despite continuing therapy. Nevertheless, imatinib has been linked to rare instances of clinically apparent acute liver injury with jaundice [12].

- Darunavir was associated with moderate-to-severe elevations in serum aminotransferase levels $(>5 \times \mathrm{ULN})$ in $3-10 \%$ of patients overall [13].

- Favipiravir is approved for influenza in Japan and umifenovir is approved for influenza in several countries. Data regarding liver effects are very limited.

However, one must keep in mind that the safety profile of a drug is not only determined by the drug's characteristics and the patient's background but also by the type of disease considered, as has been well described with sulfonamides and severe cutaneous adverse reaction in the context of HIV infection [14-18].

Since most of these drugs are susceptible to causing liver injury, we would like to remind readers of some basic principles for the characterization and definition of acute liver injury.

Acute liver injury has been internationally defined on biological criteria based on the ULN of serum alanine aminotransferase activity (ALT), serum alkaline phosphatase activity (ALP) and serum concentration of total bilirubin, as follows $[19,20]$ :

\section{- Elevation of ALT $\geq 5 \times$ ULN}

or

- Elevation of ALP $\geq 2$ ULN (in the absence of known bone pathology)

or

- Combination of an elevation of ALT $\geq 3$ ULN with simultaneous elevation of total bilirubin concentration exceeding $2 \times \mathrm{ULN}$.

Variations of these parameters below the limits set above do not constitute a DILI and the withdrawal of the medicinal product should be carried out only after all confounding factors have been excluded, especially if the expected benefit/ risk ratio is positive.

As the level of elevation of liver enzymes alone is not sufficient to reflect the severity of liver injury, we would like to remind readers of the criteria to assess the severity grading, which is based on the presence of at least one of the following [20]:

- Presence of clinical symptoms consistent with liver injury, in particular jaundice, encephalopathy and bleeding caused by coagulopathy and ascites.

- Presence of less specific symptoms such as fatigue, weakness, anorexia, nausea, vomiting, fever, chills, abdominal pain, pruritus, skin rash, etc., which have been shown to affect the likelihood of a poorer clinical outcome.

- Blood testing of total and conjugated bilirubin, and blood clotting tests (INR, Factor V and prothrombin time) should likewise be considered in the assessment of severity. For instance, Hy's law cases are considered when there is the combination of ALT $>3 \times$ ULN and total bilirubin $>2 \times \mathrm{ULN}$ in the absence of cholestasis and a more significant cause [20].

Because of the fever and pain associated with COVID-19 infection, several medicines agencies have warned physicians against the use of NSAIDs and promoted the use of acetaminophen (paracetamol) [21]. This statement could 
have resulted in a possible misuse of acetaminophen and consequently in liver injury. Moreover, some cases of major acetaminophen self-administration for prophylactic purposes have been reported and led to a special warning by the REFHEPS [22].

One should also be careful with the use of traditional medicines (alone or in association with a drug) known to increase the risk of DILI [15]. Furthermore, it has been recently suggested that nutraceuticals could increase the severity of COVID-19 infection by decreasing the natural immune defences [23]. Consequently, ANSES (The French Agency for Food, Environmental and Occupational Health and Safety) launched a warning on these products with antiinflammatory properties, including willow, birch, poplar, goldenrod, polygalas and all plants containing metabolites derived from acetylsalicylic acid like harpagophytum, Echinacea and curcuma [23].

Thus, considering all these factors, respect of the rules set out above and exclusion of confounding factors, in particular those linked to the patient's behaviour in times of crisis, should enable physicians to detect a definite DILI and to avoid inappropriate withdrawal of a potentially useful drug.

\section{Compliance with Ethical Standards}

Funding No sources of funding were used to assist in the preparation of this study.

Conflict of interest All authors have no conflicts of interest or competing interests that are directly relevant to the content of this article.

Data sharing Data sharing is not applicable to this article as no datasets were generated or analysed.

\section{References}

1. Huang C, Wang Y, Li X, et al. Clinical features of patients infected with 2019 novel coronavirus in Wuhan, China. Lancet. 2020;395(10223):497-506.

2. Guan WJ, Ni ZY, Hu Y, China Medical Treatment Expert Group for COVID-19, et al. Clinical characteristics of coronavirus disease 2019 in China. N Engl J Med. 2020. https://doi.org/10.1056/NEJMo a2002032.

3. Zhang C, Shi L, Wang F-S. Liver injury in COVID-19: management and challenges. Lancet Gastroenterol Hepatol. 2020. https:// doi.org/10.1016/S2468-1253(20)30057-1.

4. Xu L, Liu J, Lu M, Yang D, Zheng X. Liver injury during highly pathogenic human coronavirus infections. Liver Int. 2020. https:// doi.org/10.1111/liv.14435.

5. Sanders JM, Monogue ML, Jodlowski TZ, Cutrell JB. Pharmacologic treatments for coronavirus disease 2019 (COVID-19): a review. JAMA. 2020. https://doi.org/10.1001/jama.2020.6019.

6. Hydroxychloroquine LiverTox: clinical and research information on drug-induced liver injury [Internet]. Bethesda: National Institute of Diabetes and Digestive and Kidney Diseases; 2012. Hydroxychloroquine [updated 2018 Mar 25]. https://www.ncbi.nlm.nih.gov/books/ NBK548738/.
7. Azithromycin LiverTox: clinical and research information on drug-induced liver injury [Internet].Bethesda: National Institute of Diabetes and Digestive and Kidney Diseases; 2012. Azithromycin [updated 2017 Aug 8]. https://www.ncbi.nlm.nih.gov/books/NBK54 $8434 /$.

8. Interferon beta LiverTox: clinical and research information on drug-induced liver injury [Internet]. Bethesda: National Institute of Diabetes and Digestive and Kidney Diseases; 2012. Beta Interferon [updated 2018 May 4]. https://www.ncbi.nlm.nih.gov/books/NBK54 $8080 /$.

9. Lopinavir LiverTox: clinical and research information on druginduced liver injury [Internet]. Bethesda: National Institute of Diabetes and Digestive and Kidney Diseases; 2012. Lopinavir [updated 2017 Sep 1]. https://www.ncbi.nlm.nih.gov/books/NBK547961/.

10. Ritonavir LiverTox: clinical and research information on druginduced liver injury [Internet]. Bethesda: National Institute of Diabetes and Digestive and Kidney Diseases; 2012. Ritonavir [updated 2017 Sep 1]. https://www.ncbi.nlm.nih.gov/books/NBK548301/.

11. Baracitinib LiverTox: clinical and research information on druginduced liver injury [Internet]. Bethesda: National Institute of Diabetes and Digestive and Kidney Diseases; 2012. Baricitinib [updated 2018 Oct 20]. https://www.ncbi.nlm.nih.gov/books/NBK548012/.

12. Imatinib LiverTox: clinical and research information on druginduced liver injury [Internet]. Bethesda: National Institute of Diabetes and Digestive and Kidney Diseases; 2012. Imatinib [updated 2018 Apr 24]. https://www.ncbi.nlm.nih.gov/books/NBK547959/.

13. Darunavir LiverTox: clinical and research information on druginduced liver injury [Internet]. Bethesda: National Institute of Diabetes and Digestive and Kidney Diseases; 2012. Darunavir [updated 2017 Sep 1]. https://www.ncbi.nlm.nih.gov/books/NBK547994/.

14. Roujeau JC, Kelly JP, Naldi L, et al. Medication use and the risk of Stevens-Johnson syndrome or toxic epidermal necrolysis. N Engl J Med. 1995;333:1600-7.

15. Rzany B, Mockenhaupt M, Baur S, et al. Epidemiology of erythema exsudativum multiforme majus, Steven-Johnson syndrome, and toxic epidermal necrolysis in Germany (1990-1992): structure and results of a population-based registry. J Clin Epidemiol. 1996;49(7):769-73.

16. Mittmann N, Knowles SR, Koo M, Shear NH, Rachlis A, Rourke SB. Incidence of toxic epidermal necrolysis and Stevens-Johnson syndrome in an HIV cohort: an observational, retrospective case series study. Am J Clin Dermatol. 2012;13(1):49-544.

17. Taqi SA, Zaki SA, Nilofer AR, Sami LB. Trimethoprim-sulfamethoxazole-induced Steven-Johnson syndrome in an HIV-infected patient. Indian J Pharmacol. 2012;44(4):533-5.

18. Rotunda A, Hirsch RJ, Scheinfeld N, Weinberg JM. Severe cutaneous reactions associated with the use of human immunodeficiency virus medications. Acta Derm Venereol. 2003;83(1):1-9.

19. Fontana RJ, Seef LB, Andrade RJ, Björnsson E, Day CP, Serrano J, Hoofnagle JH. Standardization of nomenclature and causality assessment in drug-induced liver injury: summary of a clinical research workshop. Hepatology. 2010;52:730-42.

20. EASL. EASL clinical practice guidelines: drug-induced liver injury. J Hepatol. 2019;70:1222-611. https://doi.org/10.1016/j. jhep.2019.02.014.

21. https://www.ema.europa.eu/en/documents/press-release/ema-gives -advice-use-non-steroidal-anti-inflammatories-COVID-19_en.pdf.

22. https://afef.asso.fr/article_actu/paracetamol-et-COVID-19/.

23. https://www.anses.fr/en/content/anses-warns-against-taking-foodsupplements-could-lower-body\%E2\%80\%99s-immune-response. 